$10-1-2010$

\title{
End-of-life care for hospitalized patients with lung cancer: utilization of a palliative care service.
}

\author{
Barbara Reville, M.S., ACHPN \\ Thomas Jefferson University \\ Marshal N Miller, B.S. \\ Jefferson Medical College \\ Richard W Toner, M.S. \\ Formerly Jefferson School of Population Health \\ JoAnne Reifsnyder, Ph.D., ACHPN

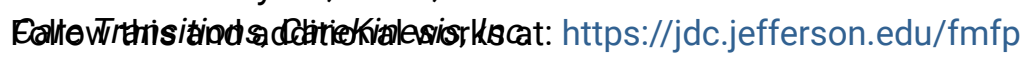 \\ Part of the Family Medicine Commons, Preventive Medicine Commons, and the Translational Medical \\ Research Commons \\ Let us know how access to this document benefits you
}

\section{Recommended Citation}

Reville, M.S., ACHPN, Barbara; Miller, B.S., Marshal N; Toner, M.S., Richard W; and Reifsnyder, Ph.D., ACHPN, JoAnne, "End-of-life care for hospitalized patients with lung cancer: utilization of a palliative care service." (2010). Department of Family \& Community Medicine Faculty Papers. Paper 23.

https://jdc.jefferson.edu/fmfp/23

This Article is brought to you for free and open access by the Jefferson Digital Commons. The Jefferson Digital Commons is a service of Thomas Jefferson University's Center for Teaching and Learning (CTL). The Commons is a showcase for Jefferson books and journals, peer-reviewed scholarly publications, unique historical collections from the University archives, and teaching tools. The Jefferson Digital Commons allows researchers and interested readers anywhere in the world to learn about and keep up to date with Jefferson scholarship. This article has been accepted for inclusion in Department of Family \& Community Medicine Faculty Papers by an authorized administrator of the Jefferson Digital Commons. For more information, please contact: JeffersonDigitalCommons@jefferson.edu. 


\title{
End-of-Life Care for Hospitalized Patients with Lung Cancer: Utilization of a Palliative Care Service
}

\author{
Barbara Reville, M.S., ACHPN, Marshal N. Miller, B.S., ${ }^{2}$ Richard W. Toner, M.S., ${ }^{3}$ \\ and JoAnne Reifsnyder, Ph.D., ACHPN ${ }^{4}$
}

\begin{abstract}
Purpose: High symptom burden and hospital mortality among patients with lung cancer argues for early palliative care intervention. Patient characteristics and discharge dispositions in hospitalized patients with lung cancer receiving usual care were compared to those referred to a new palliative care service.

Methods: A retrospective database review of all lung cancer discharges receiving usual care (UC) and palliative care service (PCS) consultation was conducted. Demographics, length of stay, discharge disposition, and mortality were described and compared. Palliative Performance Scale scores were described according to discharge disposition in the PCS group. Disposition of all patients receiving either chemotherapy or surgery was also noted.

Results: A total of 1476 hospital discharges with a diagnosis of lung cancer occurred between March 15, 2006 and June 30, 2009. Among all discharges, 9\% received chemotherapy and 29\% had surgery. The PCS was consulted for $8 \%$ of all lung cancer patients most commonly to address end-of-life-issues. PCS patients were more likely to be at the end-of-life than UC patients as evidenced by higher hospital mortality ( $31 \%$ versus $7 \%$ ), higher intensive care (ICU) mortality (67\% versus $16 \%$ ) and more frequent discharge to hospice (41\% versus $7 \%$ ). PCS patients were hospitalized a median of 6 days before a referral was made. Hospitalization was significantly longer for PCS patients $(\mathrm{M}=16.3$ days, $p<0.001)$ than $\mathrm{UC}$ patients $(\mathrm{M}=8.3$ days).

Conclusions: In the first 3 years of a new palliative care initiative consults for lung cancer patients occurred late in the hospital stay or when death was imminent.
\end{abstract}

\section{Introduction}

A S PALLIATIVE CARE PROGRAMS establish their place in the acute care hospital setting, collection and analysis of clinical, financial and operational metrics is recommended to measure impact on end-of-life care. ${ }^{1}$ At both local and national levels, such analyses permit application of national benchmarks to guide comparisons between palliative care and usual acute care. For new palliative care consultation services, data analysis permits insight into the institution's utilization of its services, patient populations most likely to benefit, practice trends and opportunities for improvement.

In this retrospective database review, characteristics and selected outcomes were compared for patients with lung cancer who received palliative care service (PCS) consultation and those who received usual care (UC). A subset of PCS and
UC patients who received care in the intensive care unit (ICU) was also compared. Lung cancer was chosen because it was the most commonly referred diagnosis and is associated with significant symptom burden at the end of life. ${ }^{2-5}$

\section{Impact of lung cancer}

In 2009 an estimated 159,390 Americans were expected to die from lung cancer, ranking it the number one cause of cancer-related death among both men and women. ${ }^{6}$ Despite progress in oncology care and patient survival for many malignancies, lung cancer mortality rates have remained high, especially among those hospitalized patients requiring critical care intervention. ${ }^{7,8}$ Chemotherapy can prolong life in these patients; however, its effect is generally limited to a few months and is associated with medical complications, quality of life issues, and costs. ${ }^{3-5,9,10}$

\footnotetext{
${ }^{1}$ Jefferson Palliative Care Service, Thomas Jefferson University, Department of Family \& Community Medicine, Philadelphia, Pennsylvania.

${ }^{2}$ Jefferson Medical College, Philadelphia, Pennsylvania.

${ }^{3}$ Formerly Jefferson School of Population Health, Philadelphia, Pennsylvania.

${ }^{4}$ Care Transitions, CareKinesis, Inc., Moorestown, New Jersey.

Accepted June 9, 2010.
} 
Common symptoms associated with lung cancer include pain, cough, dyspnea, anorexia, fatigue, hemoptysis, hiccups, depression, and emotional distress. ${ }^{3-5,10,11}$ These symptoms are present in one third to three quarters of patients with lung cancer, and typically increase in frequency and severity with disease progression. ${ }^{3-5,12}$ Unfortunately, the number of symptoms and impact on the daily lives of patients is unappreciated by their physicians. $3,10,12,13$ In one study of the characteristics and outcomes of palliative care referrals, researchers found additional symptoms, e.g., delirium, sedation, constipation, that had been neither documented nor treated by the referring team. ${ }^{12}$

\section{ICU mortality}

While mortality among patients with cancer in critical care units is not significantly different from non-cancer patients, patients with cancer are at an increased risk for morbidity from complications such as sepsis and respiratory failure. ${ }^{8}$ Inpatient mortality among patients with lung cancer who receive ICU care is as high as $60 \%$, with $43 \%$ of those patients dying while in the ICU.

\section{Benefits of palliative care consultation}

Improving palliative and end-of-life care has become a national priority. Increasingly, palliative care is viewed as integral to high-quality long-term care management across settings and illnesses regardless of prognosis. ${ }^{14}$ In 2008, the National Priorities Partnership named improving care for the dying as one of six national priorities for reforming the health care system. ${ }^{15}$ The National Comprehensive Cancer Network recommends the introduction of palliative care to all cancer patients at initial diagnosis and its integration during treatment to address symptoms and psychosocial issues. ${ }^{16}$ Other professional organizations, e.g., the American Society of Clinical Oncology, and the National Consensus Project for Quality Palliative Care are in accord with these recommendations. ${ }^{10}$ Evidence emerging from randomized controlled trials supports the effectiveness of palliative care intervention for symptom relief, patient/family satisfaction, quality of life and cost. ${ }^{17,18}$

Palliative care consultation facilitates earlier and more frequent hospice referral and lower hospital mortality. ${ }^{19-21}$ Hospice care offers an alternative to life-prolonging inpatient care and is associated with better symptom control, improved patient and family satisfaction, and feelings of respect and inclusion in care decisions. ${ }^{13,19}$ Hospice utilization by patients with lung cancer ranges from $25 \%$ to $30 \%$, a rate typical of most cancer types, yet significantly greater than patients with non-cancer diagnoses. ${ }^{22}$

The purpose of the present study was to describe the characteristics of patients with lung cancer referred to a hospital-based palliative care service and compare them to patients receiving usual care. Data on transition to hospice care and hospital mortality were compared between groups and for patients requiring ICU care, chemotherapy, or surgery. Performance scores contributed data to understand functional status and expected survival of PCS patients.

\section{Methods}

\section{Setting}

Thomas Jefferson University Hospital is an academic medical center with 700 acute care hospital beds. The PCS includes one oncology nurse practitioner with advanced palliative care certification. Four physicians (two boardcertified palliative medicine specialists, a geriatrician, and an internist), one geriatric fellow, and a music practitioner comprise the rest of the team. The inpatient consultation service began seeing patient referrals on March 15, 2006.

The PCS provided consultation to inpatient physicians only, as there is currently no palliative care or hospice unit in this institution. Referring specialties included oncology, intensive care units (includes medical, surgical, cardiac), and internal medicine, among others. Physicians consulted the PCS using an electronic ordering system that prompts a choice of 1 to 3 referral reasons from the following: advanced care planning, goals of care discussion, end-of-life issues, psychosocial distress, pain or symptom management, and mental status changes. House staff or nurse practitioners can order consults, however, the PCS routinely verifies the attending physician's consent before seeing the patient. The attending physician has declined in approximately $2 \%$ of all consults.

\section{Study design, sample, and data collection}

A retrospective review of data from the PCS and hospital administrative databases included all discharges with either a primary or secondary diagnosis of lung cancer during the study period of March 15, 2006 through June 2009. Lung cancer diagnosis was determined using ICD-9 codes. The Institutional Review Board approved this study.

Various demographic characteristics were collected on all patients including: age, gender, and ethnicity. While both databases yielded information on length of hospital stay, ICU use, chemotherapy and surgery episodes, and discharge disposition, the PCS database provided reasons for consultation, referring specialty, length of stay relative to PCS consultation (number of days before, during, and after), and performance scores.

The Palliative Performance Scale version 2 (PPS) has been validated as a measure of functional status and predictor of survival for cancer patients in hospice and inpatient palliative care settings. ${ }^{23,24}$ Scores range from 0 (death) through 100 (fully functional) in increments of $10 \%$. The PCS began routine assessment using the PPS in January 2008. Thus, performance score data were extracted from the PCS database for those patients assessed and collapsed into three categories for analysis: $20 \%$ or less, $30 \%-50 \%$, and $60 \%$ or more. These categories are consistent with an earlier analysis performed by Morita et al. ${ }^{25}$

\section{Statistical analysis}

All statistical analyses were conducted using SPSS version 15.0 (SPSS Inc., Chicago, IL). An $\alpha$ of $p<0.05$ was used to indicate significance for all statistical tests in this study.

Differences in demographics and mean length of stay were compared between the two groups using a two-tailed independent samples $t$ test. $\chi^{2}$ tests were used to compare differences in distribution of categorical variables between the groups, such as patient gender, ethnicity and disposition. Because of the wide range noted in length of hospital stay, both mean and median were calculated for comparison. For the purposes of analysis, discharge dispositions were divided into the following categories: died, hospice, skilled nursing facility/rehabilitation transfer, ventilator facility transfer, 
home, and other. The hospice category includes home hospice, inpatient hospice, or hospice care at a skilled nursing facility.

\section{Results}

\section{Patient characteristics}

There were 1476 discharges with either a primary or secondary diagnosis of lung cancer during the study period. Of these, $118(7.9 \%)$ were consulted by the PCS, leaving a sample of $1358(92 \%)$ in the usual care group. Patient characteristics for both groups are displayed in Table 1. Nine percent of all patients received chemotherapy and $29 \%$ had a surgical procedure during hospitalization.

PCS patients were significantly older $(\mathrm{M}=67.7$ years, standard deviation $[\mathrm{SD}]=12.7)$ than usual care patients $(\mathrm{M}=64.8$ years, $\mathrm{SD}=11.6, \mathrm{p}<0.01)$. There were no significant differences in gender or ethnicity between groups.

\section{Referral patterns}

The most common referring specialty was oncology, accounting for $32(27 \%)$ of the 118 lung cancer referrals to the PCS. The second and third most frequently referring specialties were the ICU $(n=30,25 \%)$ and internal medicine $(n=21$, $18 \%$ ), respectively. The most common reason for referral was end-of-life issues $(n=68,57 \%)$, followed by goals of care discussion $(n=52,44 \%)$ and advanced care planning $(n=27$, $23 \%)$. Pain and symptom management $(18 \%$ and $7 \%$, respectively) accounted for smaller percentages of referrals, with still fewer consults for psychosocial distress (4\%) and mental status changes $(3 \%)$.

\section{Length of stay, timing of PCS consultation, and discharge disposition}

The length of stay was significantly longer $(M=16.31$ days, $S D=14.61, p<0.001)$ for PCS patients than the usual care group $(\mathrm{M}=8.28$ days, $\mathrm{SD}=8.96)$. Hospitalization in both groups ranged from a minimum of 1 day to maximum of 102 days. However, median length of stay was also twice as long for PCS patients ( $\mathrm{Md}=12.5$ days) as UC patients $(\mathrm{Md}=6$ days $)$.

Table 1. Characteristics of Patients with Lung Cancer: Palliative Care Service and Usual Care Groups

\begin{tabular}{lccc}
\hline & PCS n=118 & Usual n=1358 & \\
\hline $\begin{array}{l}\text { Mean Age (SD) } \\
\text { Gender }\end{array}$ & $67.6(12.7)$ & $64.8(11.6)$ & $p=0.009$ \\
$\quad$ Male & $56(47 \%)$ & $634(47 \%)$ & NS \\
$\quad$ Female & $62(53 \%)$ & $724(53 \%)$ & \\
Ethnicity & & & \\
$\quad$ Caucasian & $72(61 \%)$ & $908(67 \%)$ & NS \\
Black & $36(31 \%)$ & $341(25 \%)$ & \\
Hispanic & $0(0 \%)$ & $6(0.4 \%)$ & \\
Asian & $6(5 \%)$ & $60(4 \%)$ & \\
$\quad$ Unknown/Other & $4(3 \%)$ & $41(3 \%)$ & \\
\hline
\end{tabular}

Percentages are rounded to the nearest whole percent.

PCS, palliative care service; SD, Standard deviation, NS, not significant.
Consults to palliative care were made a median of 6 days into lung cancer patients' hospitalization. Patients were followed by PCS for a median of 4 days, and discharged less than 2 days after consult concluded.

Discharge disposition for the PCS and usual care groups is shown in Table 2 . The PCS patients $(72 \%, n=84)$ had a higher likelihood of being at the end-of-life, i.e., discharged to hospice or died during hospital stay, than those in the usual care group $(12 \%, n=170)$.

Nearly a quarter of the patients in each group received ICU care and $20 \%$ of these patients died during the admission (Table 3). However, more PCS patients in the ICU died $(67 \%$ versus $16 \%$ ) or were discharged to hospice (17\% versus $4 \%$ ), compared to the usual care group.

Of all hospitalized patients receiving chemotherapy, 8\% died or enrolled in hospice. For patients who had surgery, $11 \%$ died or were discharged to hospice.

\section{Palliative Performance Scale scores}

Palliative Performance Scale scores were available for 55 (46.6\%) patients seen by PCS. Overall, $85 \%$ of patients scored at or below $50 \%$. The discharge disposition of PCS patients according to PPS score is presented in Table 4 . Patients with a PPS score of $20 \%$ or less most often died in the hospital $(67 \%, n=10)$. Patients with a PPS score from $30 \%$ to $50 \%$ were most commonly discharged to hospice $(53 \%, n=17)$.

\section{Discussion}

This study depicts utilization of a palliative care team during its early implementation period and may be instructive for others pioneering similar efforts. However, a clear picture of the success or challenge of palliative care's integration is limited because existing data sources only partially reveal the unmet need among usual care patients.

This hospital database did not provide data on symptom severity, performance status, or disease stage metrics to identify usual care patients with palliative care needs. ${ }^{1}$ However, it did permit identification of patients who received chemotherapy or surgery during their hospital stay, a possible indicator of better performance status and ineligibility for palliative care consultation. If this cohort were eliminated from the sample on that basis, the PCS was involved in a larger percentage, i.e., $13 \%$ versus $8 \%$, of all eligible patients with lung cancer. Ultimately, this variable was invalidated as a marker of palliative care ineligibility when disposition data revealed that $19 \%$ of those who received chemotherapy or surgery died or chose hospice. While the use of palliative chemotherapy can offer some benefit without severe toxicity, these data raise several questions about appropriate goals and use of health care resources that are worthy of future research. $^{26}$

The PCS was utilized for a small percentage of patients with lung cancer and when used, was consulted late in the course of hospitalization. Most patients referred died or were discharged to hospice. These referral patterns reveal a common misperception among providers that palliative care is mutually exclusive with life-prolonging care, frequently justified by the response that their patient is "not ready 
Table 2. Discharge Disposition: Palliative Care Service versus Usual Care Groups

\begin{tabular}{lccccrrr}
\hline & Died & Hospice & SNF/Rehab & Ventilator facility & Home & Other & Total \\
\hline Usual & $84(6)$ & $86(6)$ & $180(13)$ & $0(0)$ & $975(72)$ & $33(2)$ & $1358(100)$ \\
PCS & $36(31)$ & $48(41)$ & $10(8)$ & $3(3)$ & $20(17)$ & $1(1)$ & $118(100)$ \\
Total & $120(8)$ & $134(9)$ & $190(13)$ & $3(<1)$ & $995(67)$ & $34(2)$ & $1476(100)$ \\
\hline
\end{tabular}

Numbers in parentheses represent percent of row total. Percentages are rounded to the nearest whole percent.

PCS, palliative care service; SNF, skilled nursing facility.

yet." $^{10(\mathrm{p} 408 \mathrm{~S})}$ Late referrals do not prove palliative care needs were unmet, but may reflect providers' comfort with addressing palliative care needs without specialty referral before the end of life. Other explanations for nonreferral or late referrals may include lack of familiarity with palliative care among providers, insufficient or ineffective marketing, or refusal by patients or their families.

Hospital data are not available to permit comparisons of symptom prevalence and severity between groups. Patients were referred to PCS for end-of-life issues twice as often as for pain or symptom management despite evidence of high rates of pain and other symptoms in this population. ${ }^{3-5,12}$ However, referrals for psychosocial distress or mental status changes were uncommon. Usual care patients may have received symptom palliation from the primary hospital team, the hospital pain service or psychosocial providers. However, previous research suggests that patients' symptoms are often underrecognized and undertreated. ${ }^{3,12,13}$

Studies using a variety of designs for measuring clinical metrics such as pain and symptom scores, patient satisfaction, ICU mortality, or costs of care have shown benefits of inpatient palliative care teams. ${ }^{12,17,18,21}$ The present study does not report prevalence and severity of pain and symptoms or the success of the PCS at ameliorating these complications of lung cancer. However, further standardization of symptom assessment is a goal for the PCS.

The PCS facilitated higher rates of hospice referral compared to usual care. The strength of these conclusions is limited by the sample sizes for the PCS group and the ICU subgroup, as well as inability to control for disease stage, illness acuity, or comorbidities.

Table 3. Discharge Disposition Among Palliative Care and Usual Care Groups Receiving INTENSIVE CARE Unit CARE

\begin{tabular}{|c|c|c|}
\hline Disposition & $\begin{array}{l}\text { Palliative } \\
\text { care group }\end{array}$ & $\begin{array}{c}\text { Usual } \\
\text { care group }\end{array}$ \\
\hline Died & $20(67)$ & $51(16)$ \\
\hline Hospice & 5 (17) & $12(4)$ \\
\hline $\begin{array}{l}\text { Skilled nursing or } \\
\text { rehabilitation facility }\end{array}$ & $1(3)$ & $75(24)$ \\
\hline Ventilator facility & $3(10)$ & $0(0)$ \\
\hline Home & $1(3)$ & $173(55)$ \\
\hline Other & $0(0)$ & $5(2)$ \\
\hline Total & $30(100)$ & $317(100)$ \\
\hline
\end{tabular}

Numbers in parentheses represent percent of column total rounded to nearest whole percent.
The Palliative Performance Scale scores in the PCS sample were consistent with scores among seriously ill patients in other studies. ${ }^{23-25}$ Overall, $85 \%$ of patients scored at or below $50 \%$, a score that is associated with a high likelihood of death in 6 months thereby connoting hospice eligibility. ${ }^{23}$ Scores were less than $20 \%$ in more than one quarter of cases, predicting survival of 1 week. The majority of these patients died in hospital whereas those with scores that fell in the $30 \%-50 \%$ range were most often discharged with hospice, indicating some expected survival beyond hospital discharge. An inpatient hospice unit in this hospital would support the sickest patients with comfort-based care and family-focused services. These institutional transfers would open acute care beds and resources, especially in ICU, for patients more likely to benefit.

The findings of the present study, although limited by the relatively small PCS sample, support previous findings of high mortality rates in patients with lung cancer who receive critical care. ${ }^{7,8}$ Overall, inpatient mortality among patients receiving ICU care was $20 \%$ in this study. However, mortality among PCS patients was higher (67\%). More analysis is needed to determine reasons for this disparity, which previous research has suggested may be a result of unaccounted patient and hospital characteristics. ${ }^{27}$

The retrospective design and disparate group sizes limit this study's conclusions about both the effectiveness of the PCS and whether certain markers, such ICU care or low PPS scores are accurate predictors of mortality. It is also likely, that since the PCS is relatively new, and referral practices are evolving, that these data do not reflect more recent referral trends. Nonetheless, the findings sufficiently support evidence of gaps in end-of-life care for patients with lung cancer to warrant further initiatives by our nascent palliative care team. Planned revisions in processes of care will include routine data collection on symptom assessment, interventions, and outcomes of PCS referrals, e.g., pain and symptom scores, establishment of patient's goals of care, transition away from life-prolonging technology. Patient, family, and provider satisfaction with PCS services will further inform these efforts.

Education of all providers on the complex care needs of lung cancer patients, the scope of palliative care services, and its potential benefits is needed. The use of referral criteria for palliative care consultation can serve as both an education, screening, and marketing tool and will be the aim of a future performance improvement project. Development of a longterm care management model in collaboration with lung cancer care providers has the potential to promote earlier case finding. ${ }^{17}$ Continued efforts to integrate the PCS earlier and more frequently for patients with chronic and/or life-threatening 
Table 4. Discharge Disposition by Palliative

Performance Scale Scores in Palliative CARE Group

\begin{tabular}{lcclr}
\hline & \multicolumn{5}{c}{ Palliative Performance Score } \\
\hline Disposition & $\leq 20 \%$ & $30-50 \%$ & $\geq 60 \%$ & Total \\
$\quad$ Died & $10(67)$ & $4(13)$ & $0(0)$ & $14(25)$ \\
Hospice & $1(7)$ & $17(53)$ & $2(25)$ & $20(36)$ \\
SNF/Rehab & $1(7)$ & $4(13)$ & $2(25)$ & $7(13)$ \\
Ventilator & $2(13)$ & $0(0)$ & $0(0)$ & $2(4)$ \\
$\quad$ Facility & & & & \\
Home & $1(7)$ & $7(22)$ & $4(50)$ & $12(22)$ \\
Total & $15(100)$ & $32(100)$ & $8(100)$ & $55(100)$ \\
\hline
\end{tabular}

Numbers in parentheses represent percent of column total. Percentages are rounded to the nearest whole percent. PPS routinely recorded starting in 2008 .

PPS, Palliative Performance Scale; SNF, skilled nursing facility.

illnesses, particularly those with high symptom burden, is consistent with the purpose of palliative care and supports national goals to improve end-of-life care. ${ }^{15,28}$

\section{Acknowledgments}

Thanks are extended to Abbie J. Santana, MSPH, and James Diamond, PhD for guidance in statistical analysis. We are also grateful to the Jefferson Palliative Care Service who pioneered palliative care at this institution: Dr. Christine Arenson, Dr. David Axelrod, Dr. Susan Parks, Dr. Danielle Snyderman, Dr. Rebecca Maury, and Ms. Linda Ferguson, CRNP.

\section{Author Disclosure Statement}

No competing financial interests exist.

\section{References}

1. Weissman DE, Meier DE, Spragens LH: Center to Advance Palliative Care palliative care consultation service metrics: Consensus recommendations. J Palliat Med 2008;11:12941298.

2. Silvestri GA, Sherman C, Williams T, Leong S, Flume P, Turrisi A: Caring for the dying patient with lung cancer. Chest 2002;122:1028-1036.

3. Podnos YD, Borneman TR, Koczywas M, Uman G, Ferrell BR: Symptom concerns and resource utilization in patients with lung cancer. J Palliat Med 2007;10:899-903.

4. Temel JS, Pirl WF, Lynch TJ: Comprehensice symptom management in patients with advanced-stage non-small-cell lung cancer. Clinical Lung Cancer 2006;7:241-249.

5. Lutz S, Norrell R, Bertucio C, Kachnic L, Johnson C, Arthur D, Schwarz M, Palardy G: Symptom frequency and severity in patients with metastatic or locally recurrent lung cancer: A prospective study using the Lung Cancer Symptom Scale in a community hospital. J Palliat Med 2001;4:157-165.

6. Jemal A, Siegel R, Ward E, Hao Y, Xu J, Thun MJ: Cancer statistics, 2009. CA Cancer J Clin 2009;59:225-249.

7. Reichner CA, Thompson JA, O'Brien S, Kuru T, Anderson ED: Outcome and code status of lung cancer patients admitted to the medical ICU. Chest 2006;130:719-723.
8. Taccone FS, Artigas AA, Sprung CL, Moreno R, Sakr Y, Vincent J: Characteristics and outcomes of cancer patients in European ICUs. Crit Care 2009;13:10.

9. Marino P, Pampallona S, Preatoni A, Cantoni A, Invernizzi F: Chemotherapy vs. supportive care in advanced nonsmall-cell lung cancer. Chest 1994;106:861-865.

10. Griffin JP, Koch KA, Nelson JE, Cooley ME: Palliative care consultation, quality-of-life measurements, and bereavement for end-of-life care in patients with lung cancer: ACCP evidence-based clinical practice guidelines (2nd edition). $\underline{\text { Chest }}$ 2007;132:404S-422S.

11. Marinella, M.A.: Diagnosis and management of hiccups in the patient with advanced cancer. J Support Oncol 2009;7:122-127.

12. Braiteh F, El Osta B, Palmer JL, Reddy SK, Bruera E: Characteristics, findings, and outcomes of palliative care inpatient consultations at a comprehensive cancer center. J Palliat Med 2007;10:948-955.

13. Von Gunten CF: Interventions to manage symptoms at the end of life. J Palliat Med 2005;8:S88-S94.

14. Meier DE, Casarett DJ, von Gunten CF, Smith WJ, Storey CP: Palliative medicine: Politics and policy. I Palliat Med 2010;13:1-6.

15. National Priorities Partnership: Aligning Our Efforts to Transform America's Healthcare. National Priorities and Goals. Washington, D.C.: National Quality Forum, 2008.

16. National Comprehensive Cancer Network. Clinical practice guidelines in oncology. Palliative care, 2009. www.ncen .org/professionals/physician_gls/PDF/palliative.pdf (Last accessed December 29, 2009).

17. Bakitas M, Lyons KD, Hegel MT, Balan S, Brokaw FC, Seville J, Hull JG, Li Z, Tosteson TD, Byock IR, Ahles TA: Effects of a palliative care intervention on clinical outcomes in patients with advanced cancer. JAMA 2009;302:741-749.

18. Morrison RS, Penrod JD, Cassel JB, Caust-Ellenbogen M, Litke A, Spragens L, Meier DE: Cost savings associated with US hospital palliative care consultation programs. Arch Intern Med 2008;168:1783-1790.

19. Fromme EK, Bascom PB, Smith MD, Tolle SW, Hanson L, Hickam DH, Osborne ML: Survival, mortality, and location of death for patients seen by a hospital-based palliative care team. I Palliat Med 2006;9:903-911.

20. Gade G, Venohr I, Conner D, McGrady K, Beane J, Richardson RH, Williams MP, Liberson M, Blum M, Della Penna R: Impact of an inpatient palliative care team: A randomized controlled trial. J Palliat Med 2008;11:180-190.

21. Elsayem A, Smith ML, Parmley L, Palmer JL, Jenkins R, Reddy S, Bruera E: Impact of a palliative care service on inhospital mortality in a comprehensive cancer center. I Palliat Med 2006;9:894-902.

22. Iwashyna TJ, Zhang JX, Christakis NA: Disease-specific patterns of hospice and related healthcare use in an incidence cohort of seriously ill elderly patients. J Palliat Med 2002;5:531-538.

23. Harrold J, Rickerson E, Carrol JT, McGrath J, Morales K, Kapo J, Casarett D: Is the Palliative Performance Scale a useful predictor of mortality in a heterogeneous hospice population? J Palliat Med 2005;8:503-509.

24. Lau F, Downing M, Lesperance M, Shaw J, Kuziemsky C: Use of Palliative Performance Scale in end-of-life prognostication. J Palliat Med 2006;9:1066-1075.

25. Morita T, Tsunoda J, Satoshi I, Satoshi C: Validity of the Palliative Performance Scale from a survival perspective. I Pain Symptom Manage 1999;18:2-3. 
26. Harrington SE, Smith TJ: The role of chemotherapy at the end of life: "When is enough, enough?" JAMA 2008;299: 2667-2678.

27. Knaus WA, Wagner DP, Zimmerman JE, Draper EA: Variations in mortality and length of stay in intensive care units. Ann Intern Med 1993;118:753-761.

28. National Consensus Project for Quality Palliative Care: Clinical Practice Guidelines for Quality Palliative Care, 2nd ed. Pittsburgh, PA: 2009.
Address correspondence to: Barbara Reville, M.S., ACHPN Jefferson Palliative Care Service Thomas Jefferson University Department of Family $\mathcal{E}$ Community Medicine

1015 Walnut Street, Suite 401 Philadelphia, PA 19107

E-mail: Barbara.Reville@jefferson.edu 
This article has been cited by:

1. Christina L. Bell, Meiko Kuriya, Daniel Fischberg. 2011. Hospice Referrals and Code Status: Outcomes of Inpatient Palliative Care Consultations Among Asian Americans and Pacific Islanders with Cancer. Journal of Pain and Symptom Management . [CrossRef] 